\title{
Similarities in stress physiology among patients with chronic pain and headache disorders: evidence for a common pathophysiological mechanism?
}

\author{
Rune Bang Leistad · Kristian Bernhard Nilsen • \\ Lars Jacob Stovner · Rolf Harald Westgaard • \\ Magne Rø $\cdot$ Trond Sand
}

Received: 30 January 2008 / Accepted: 1 March 2008/Published online: 29 March 2008

(C) Springer-Verlag 2008

\begin{abstract}
One common feature of chronic musculoskeletal pain and headaches are that they are both influenced by stress. Among these, tension-type headache (TTH), fibromyalgia (FMS) and chronic shoulder/neck pain (SNP) appear to have several similarities, both with regard to pathophysiology, clinical features and demographics. The main hypothesis of the present study was that patients with chronic pain (TTH, FMS and SNP) had stress-induced features distinguishing them from migraine patients and healthy controls. We measured pain, blood pressure, heart rate (HR) and skin blood flow (BF) during (1 h) and after (30 min) controlled low-grade cognitive stressor in 22 migraine patients, $18 \mathrm{TTH}$ patients, 23 FMS patients, 29 SNP patients and 44 healthy controls. FMS patients had a lower early HR response to stress than migraine patients, but no differences were found among FMS, TTH and SNP patients. Finger skin BF decreased more in FMS patients compared to migraine patients, both during and after the test. When comparing chronic pain patients (chronic TTH, FMS and SNP) with those with episodic pain (episodic TTH and migraine patients) or little or no pain (healthy controls), different adaptation profiles were found during
\end{abstract}

R. B. Leistad ( $₫)$ - K. B. Nilsen · L. J. Stovner · M. Rø ·

T. Sand

Department of Neuroscience, Norwegian University of Science and Technology, 7489 Trondheim, Norway

e-mail: rune.leistad@ntnu.no

R. H. Westgaard

Department of Industrial Economics and Technology

Management, Norwegian University of Science and Technology,

7006 Trondheim, Norway

R. B. Leistad · K. B. Nilsen · L. J. Stovner · M. Rø · T. Sand St Olavs Hospital Trondheim University Hospital,

7489 Trondheim, Norway the test for systolic and diastolic blood pressure, HR and skin BF in the chronic group. In conclusion, these results suggest that TTH, FMS and SNP patients may share common pathophysiological mechanisms regarding the physiological responses to and recovery from low-grade cognitive stress, differentiating them from episodic pain conditions such as migraine

Keywords Tension-type headache (TTH) - Migraine . Fibromyalgia (FMS) $\cdot$ Chronic neck/shoulder pain (SNP) . Stress $\cdot$ Chronic pain

\section{Introduction}

Chronic musculoskeletal pain and headaches are highly prevalent and they represent major health problems in most industrialised countries [1,2]. Among such pain conditions tension-type headache (TTH) is the most prevalent primary headache [2], while fibromyalgia (FMS) is a common and poorly understood cause of sick leave and disability in western countries [3]. Different pain syndromes of unknown aetiology often share clinical features $[4,5]$ and several studies indicate that FMS and TTH have several common features, both with regard to pathophysiology, demographics and clinical symptoms, though there are also clear differences between the two conditions $[6,7]$. One common feature for all these conditions is the patient's reports of stress as a trigger for the development of pain [8, 9]. Mental stress and especially stressful work situations have been related to development of pain in both musculoskeletal pain and headache [10-17].

Although there are many studies linking mental stress to headache and musculoskeletal pain, little solid evidence exists on the potential mechanisms for this. However, there 
are several theoretical models describing how stress may lead to disease and subjective complaints such as pain. One common denominator for these models is that they emphasise the lack of physiological recovery after stress as a risk factor for development of pain or other diseases [1822]. Although studies investigating the physiological validity of these theoretical models are sparse [23], there are some interesting findings. Reported "need for recovery after work" can increase the risk for cardiovascular disease [24] and increase the risk for future sick leave [25]. Work stress has also been linked to lower evening cortisol levels, interpreted as a sign of lack of recovery [26]. Elevated blood pressure (BP) or peripheral vasoconstriction after stressful work may be a good indicator of prolonged arousal after stress, as our group has demonstrated that BP and finger skin blood flow (BF) responses to a stressful task lasts longer than other physiological stress responses [27]. Sustained activation of physiological stress responses may potentially lead to a reduced dynamic capacity to respond to new stressors [28]. Reduced physiological stress responses to different stressors have been found in both FMS and TTH, though results are conflicting [29-31].

We had previously studied the physiological and biochemical effects of experimentally induced cognitive stress on patients with migraine and TTH [17, 32, 33], and on patients with FMS and chronic shoulder/neck pains (SNP) compared to controls [16, 34]. As many of the reported features in these diseases are overlapping, it was of interest to compare different pain syndromes of unknown aetiology to identify potential similarities and differences both in response and recovery to stress. The main hypothesis of the study was that patients with chronic pain (chronic TTH, FMS and SNP) had autonomic activity during and after a stressful task, distinguishing them from patients with less regular pain (migraine or episodic TTH) and healthy controls that have little or no pain.

\section{Methods}

\section{Subjects}

Totally, 44 healthy control subjects [ 35 women (mean age 39.7 years) and 9 men (36.6 years)] and 92 patients belonging to one of four diagnostic groups participated in this study. A total of 22 patients had migraine, 20 women (39.8 years) and 2 men (45.0 years), and 13 of these patients had aura preceding the headache. Eighteen patients had TTH, including nine women (33.8 years) and nine men (35.7 years). Twelve of the TTH patients had chronic TTH (daily headache) and six had episodic TTH (three patients reported 4-7 headache days per month and three patients reported 7-14 headache days per month). A total of 23 female patients were diagnosed with FMS (48.3 years), and 29 female patients (41.1 years) had SNP. Detailed subject and headache history data are shown in Table 1 (one migraine patient reported some attacks of short duration). Patients were diagnosed after an interview and physical examination by a neurologist or a specialist in physical medicine and rehabilitation. Headache diagnoses were made according to the International Headache Society classification of headache from 1988 [35]. Migraineurs with TTH more than 7 days per month were excluded. FMS patients were included if they fulfilled the 1990 American College of Rheumatology criteria (ACR criteria) for FMS [36]. SNP patients were included if they reported SNP (more than 3 months during previous year) with local tenderness or pain. SNP patients were included even if they reported pain in other body regions, as long as pain in the shoulder and neck region was their main problem. No SNP patients fulfilled the ACR criteria for FMS.

Control subjects did not suffer from headache or musculoskeletal pain for more than 1 day per month. Exclusion criteria were: neoplastic disease, hypertension, infectious disease, metabolic, endocrine or neuromuscular diseases, significant psychiatric disorders, connective tissue disorder, tendinitis, recent significant accident or injury, pregnancy, daily medication with neuroleptics, antiepileptics, $\mathrm{Ca}^{2+}$ blockers, $\beta$-blockers, antidepressants and significant associated diseases affecting either the heart, lungs, cerebrovascular system, or the central or peripheral nervous system. The project was approved by the regional ethics committee. All participants gave written informed consent and received NOK 500 (USD 75) for transport expenses and inconvenience. The participants were provided with written information concerning the aim of the study prior to the day of the stress test. The aim of studying pain and headache was mentioned, but the information focused on the practical details of the procedure. Upon arrival on the test day, subjects went through a structured interview concerning headaches and musculoskeletal complaints (distribution, severity and duration). These data have been presented elsewhere and are not included in the present study [16, 17, 32-34].

Physiological recordings

Autonomic activity was measured indirectly by continuous recording of non-invasive finger BP (Portapres, TNO Biomedical Instrumentation, Amsterdam, The Netherlands) [37] and skin BF in the thumbs (Moorlab, time constant $0.02 \mathrm{~s}$, low-pass filter $22 \mathrm{kHz}$; Moor Instruments Ltd., Devon, England). The BP cuffs were mounted on the intermediate phalanx on the left middle and ring fingers. Finger skin $\mathrm{BF}$ was measured bilaterally with the electrodes (fibre separation $0.5 \mathrm{~mm}$ ) placed on the volar side of 
Table 1 Background data of subjects included in the study

\begin{tabular}{lcll}
\hline Subject group & $\begin{array}{l}\text { Gender } \\
\text { ratio (F:M) }\end{array}$ & $\begin{array}{l}\text { Mean age } \\
\text { (range) }\end{array}$ & $\begin{array}{l}\text { Mean general } \\
\text { tension (VAS) (range) }\end{array}$ \\
\hline Controls $(n=44)$ & $35: 9$ & $39.0(19-61)$ & $25.8(0-84)$ \\
Migraine $(n=22)$ & $20: 2$ & $40.2(20-60)$ & $35.1(1-87)$ \\
Tension-type headache $(n=18)$ & $9: 9$ & $34.7(19-52)$ & $25.1(0-65)$ \\
Fibromyalgia $(n=23)$ & $23: 0$ & $48.3(32-63)$ & $47.3(0-100)$ \\
Chronic shoulder/neck pain $(n=29)$ & $29: 0$ & $41.1(19-59)$ & $43.4(8-89)$ \\
Chronic pain patients $(n=64)$ & $59: 4$ & $42.9(19-63)$ & $42.1(0-100)$ \\
Episodic pain patients/healthy controls $(n=72)$ & $57: 15$ & $38.7(19-61)$ & $28.0(0-87)$ \\
\hline
\end{tabular}

the distal phalanx (pulp) of the thumbs. The average from the left and right thumb was used for analysis, because a significant side difference was not found. Signals were sampled at $200 \mathrm{~Hz}$. Heart rate (HR) and BP were calculated with the Beatscope 1.0 software (TNO, Amsterdam, The Netherlands). Muscular activity was recorded with surface electromyography (EMG) bilaterally in the trapezius, splenius, temporalis and frontalis muscles, as described in a previous paper [16, 17]. Because we previously did not find differences in muscular activity between the controls and patients, these variables were not included in the statistical analyses in the present study. Respiration was recorded with a thermistor (Embla S-AF-010, Flaga, Reykjavik, Iceland) below the nose with active elements in each nostril and in front of the mouth, but respiration frequency was not analysed in this study due to technical difficulties (seven controls, eight migraine patients, two TTH patients, one FMS patient and two SNP patients had corrupted respiration rate data).

\section{Procedure}

The subjects were seated in an ordinary office chair without armrests and performed a two-choice reaction-time test presented on a PC monitor for $60 \mathrm{~min}$ [38]. The test involved a grid (seven columns by five rows) in which a large and a small square were placed randomly [39]. The subject was then presented with a suggestion on how to move the small square to superimpose it on the large square (for instance, "two up, four right"), and the subjects responded by pressing either "right" or "wrong" on a panel before them with their right index or ring finger, respectively. Then the positions of the squares were changed and a new suggestion was displayed. The subjects were instructed to carry out the assignment as fast and correctly as possible, and the computer provided feedback on performance by informing whether the answer was correct or not and how fast the trial was performed (very slow, slow, normal, fast or very fast) [40]. The "normal" response for each subject was determined as the mean response time during a 5-min trial period. The subjects were acclimated to the lab environment for $30 \mathrm{~min}$, during which the procedure was explained and the recording equipment was mounted. The recording started with 5 min uninstructed rest (UIR, baseline) followed by $5 \mathrm{~min}$ active, instructed rest with visual EMG feedback (FB). FB data were not included in the statistical analysis because we believe that UIR probably is a more realistic "real-life" baseline. The cognitive task was then performed for $1 \mathrm{~h}$ (800-1,500 trials), followed by $30 \mathrm{~min}$ recording during rest (recovery period). The subjects were asked to relax while seated and to move as little as possible during the recovery period. After the baseline and FB periods, at 10min intervals during the cognitive task, and at 10-min intervals during the recovery period, the subjects were asked to mark on a $100 \mathrm{~mm}$ visual analogue scale (VAS) their level of pain with endpoints "no pain" and "worst imaginable pain". Pain was reported bilaterally in the trapezius, splenius, temporalis and frontalis areas. No patient had to be excluded because of headache attacks during the test. Venous blood was sampled before the test (immediately after the interview was concluded) and immediately after the stress period (after $60 \mathrm{~min}$ ). Blood sample data will not be reported in this paper.

Some subjects had partly missing data due to technical difficulties: two controls, two migraine patients and four SNP patients had corrupted BP and HR data during the test and recovery period, and one patient with TTH had corrupted $\mathrm{BP}, \mathrm{HR}, \mathrm{BF}$ and pain data during the recovery period.

\section{Data analysis}

Mean values for systolic blood pressure (SBP), diastolic blood pressure (DBP), HR and finger BF were calculated for the UIR period, and for each 10-min interval throughout the stressful task and recovery period. The pain variable used was defined as the maximal pain reported at the eight muscle locations (trapezius, splenius, temporalis and frontalis muscles; left and right side) at each 10-min interval. These data were used in statistical ANOVA models. 
Baseline values were compared among the groups with Student's two-group $t$ test. Repeated measures ANOVA time $\times$ group interaction was used to explore differences in response and recovery patterns between groups. It was the potential differences in response and recovery patterns to the stressor, which was of interest in the present study, and not differences in absolute levels, hence we report time $\times$ group interaction, $F$ and $P$ values. Three different models with selected dependent variables were applied to explore different parts of the stress response and recovery profile. To examine how the novelty of the stressor influenced the subjects, the first $10 \mathrm{~min}$ and the baseline were compared in an $F_{2}$-model [SBP, DBP, HR and BF: $y=$ (baseline (UIR), 0-10 $\mathrm{min})$; pain: $y=(0,10 \mathrm{~min})]$. This was described as the early (acute) stress response. After the first $10 \mathrm{~min}$, it was assumed that the novelty aspect of the stressor was gone, and we examined how the subjects adapted to the stressor with an $F_{6}$ model with six repeated dependent variables [SBP, DBP, HR and BF: $y=(0-10, \quad 10-20, \quad 20-30,30-40,40-50,50-60 \mathrm{~min})$; pain: $y=(10,20,30,40,50,60 \mathrm{~min})]$ to examine how the subjects adapted to the stressor. This was described as the adaptation response to stress. An $F_{3}$-model with three dependent variables $[y=(65-75,75-85,85-95 \mathrm{~min})]$ was used to examine how the subjects recovered from the stressor. For pain, recovery was evaluated with an $F_{4^{-}}$ model with four dependent variables $[y=(65,75,85$, $95 \mathrm{~min}$ ). An $F_{4}$-model instead of an $F_{3}$-model was necessary for the pain variable to cover the same time frame as the physiological variables, since pain was measured every $10 \mathrm{~min}$ and not as an average of continuously measured 10-min intervals. The ANOVA models were corrected for non-sphericity by reduced degrees of freedom with Huyhn-Feldts method. In the present study, we report differences in migraine and TTH patients compared to FMS and SNP patients. Differences in TTH patients compared to migraine patients and healthy controls, and differences in FMS patients compared to SNP patients and healthy controls have been reported in previous papers [16, 17, 32-34].

As FMS and SNP and, to some extent, TTH are associated with chronic pains, and based on observations made in earlier studies, it was of interest to compare subjects with chronic pain (12 chronic TTH, FMS and SNP patients) against subjects with episodic or no pain (controls, migraine and six episodic TTH patients). The basis for this grouping was that the episodic pain patients are pain-free most of the time. It was also of interest to investigate whether headache patients as a group were different from patients with musculoskeletal pains. Therefore, $F_{2}, F_{6}$ and $F_{3} / F_{4}$ analyses were performed both on chronic pain patients versus non-chronic/healthy subjects, and on headache patients versus musculoskeletal pain patients.

As our general statistical strategy involves a large number of comparisons, some might argue that there is a need for a multiple-comparison adjustment to control for type I errors. We chose not to do this, as this would create other problems, such as an increase in type II errors [41, 42]. Also, as the study was considered to be mainly hypothesis-generating and not so much hypothesis-controlling, we believe that findings worthy of further research might be missed by applying too rigid criteria to the statistical analyses. A two-tailed significance level of $<0.05$ was considered significant in the statistical analyses. $P$ values within a range of $0.05-0.10$ were defined as trends.

\section{Results}

Comparison of baseline, early test response patterns and test adaptation

There were no differences in SBP, DBP, HR or BF baseline values among the groups $(P \geq 0.149$; Fig. 1a-d). Baseline pain was found to be higher in FMS patients compared to migraine patients $(P<0.0005$; Fig. 1e) and TTH patients $(P=0.037)$ and tended to be higher in SNP patients compared to migraine patients $(P=0.054)$. When comparing the early response patterns $\left(F_{2} ;\right.$ Table 2; Fig. 1a-e), a significant difference in early $\mathrm{HR}$ response caused by a lower initial increase in HR was found in FMS compared to migraine patients $\left(F_{2}=7.33\right.$, $P=0.01$; Fig. 1c), while no difference was found between FMS and TTH.

Migraine patients were significantly different from FMS patients with less increase in vasoconstriction measured by finger $\mathrm{BF}$ during the test $\left(F_{6}=3.43, P=0.021\right.$; Table 2 and Fig. 1d), while the difference between TTH and FMS patients was not significant. We also found a trend towards a different SBP adaptation during the test in migraine patients compared to FMS patients $\left(F_{6}=2.23, P=0.079\right.$; Fig. 1a), with a continued SBP increase in FMS while migraine patients stabilized or dropped slightly in SBP. The corresponding difference between TTH and FMS was not significant. It was also found that TTH patients had a larger increase in pain compared to FMS $\left(F_{6}=3.25, P=0.025\right)$ and SNP patients $\left(F_{6}=3.10, P=0.033\right.$; Fig. $\left.1 \mathrm{E}\right)$.

\section{Comparison of recovery patterns}

Looking at the recovery patterns ( $F_{3}$ and $F_{4}$; Table 2$)$, we found a significantly less decreasing $\mathrm{BF}$ recovery profile in 

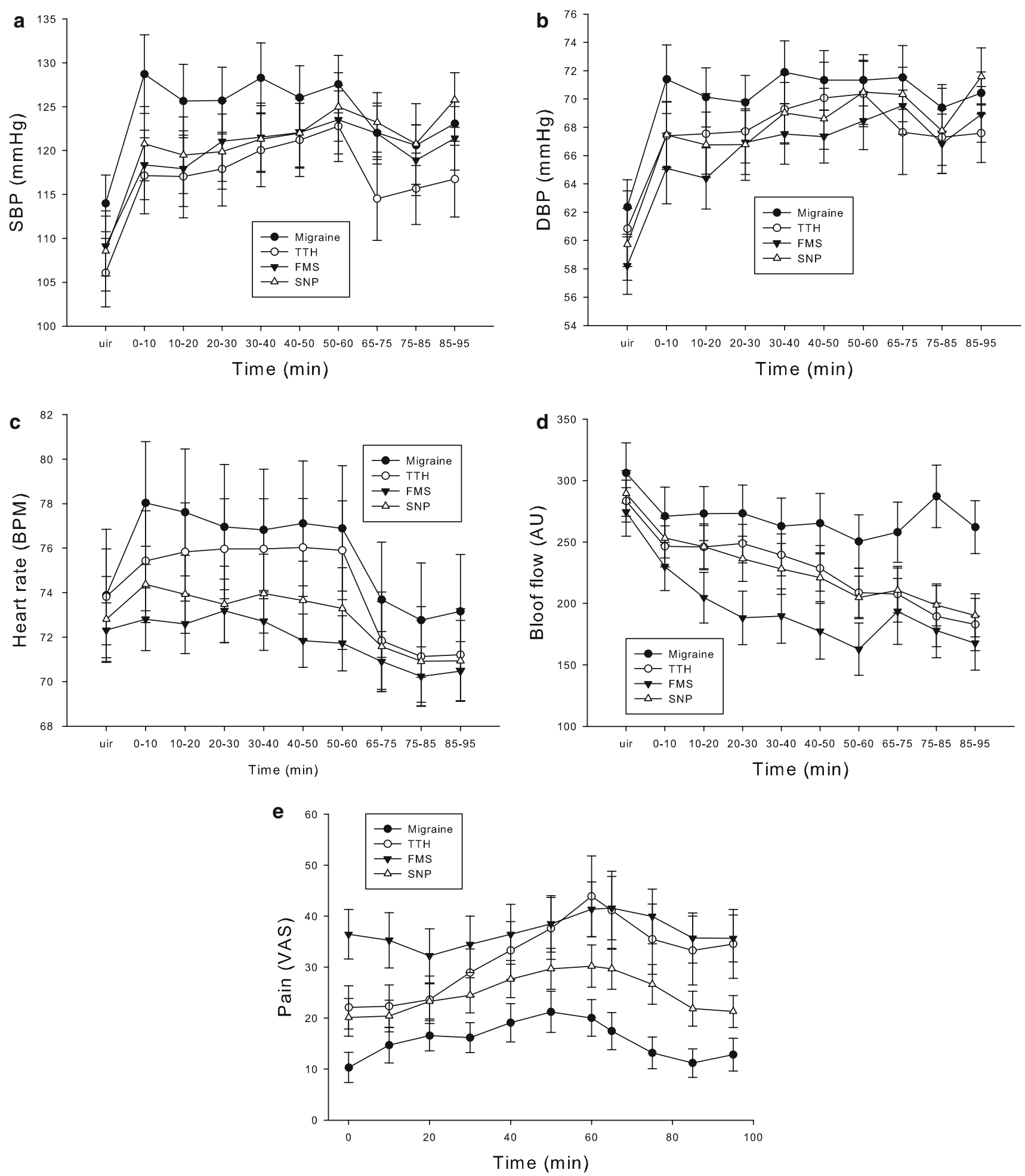

Fig. 1 Development of systolic BP (a), diastolic BP (b), heart rate (c) finger blood flow (d) and pain (e) throughout the stressful task and recovery period in patients with migraine, TTH, FMS and SNP.
Values are given as group means (SEM). UIR: uninstructed rest period (baseline EMG). 0-60 min: duration of the cognitive stressful task. 65-95 min: relaxation period after the test migraineurs compared to SNP patients $\left(F_{3}=4.99\right.$, $P=0.01$, Fig. 1d), and a similar trend towards a difference in migraineurs compared to FMS patients $\left(F_{3}=3.27\right.$,
$P=0.05)$. Other cardiovascular and pain recovery patterns in TTH patients were similar to FMS and SNP patients $\left(F_{3}\right.$, $4 \leq 1.44, P \geq 0.24$; Fig. 1a-e). 


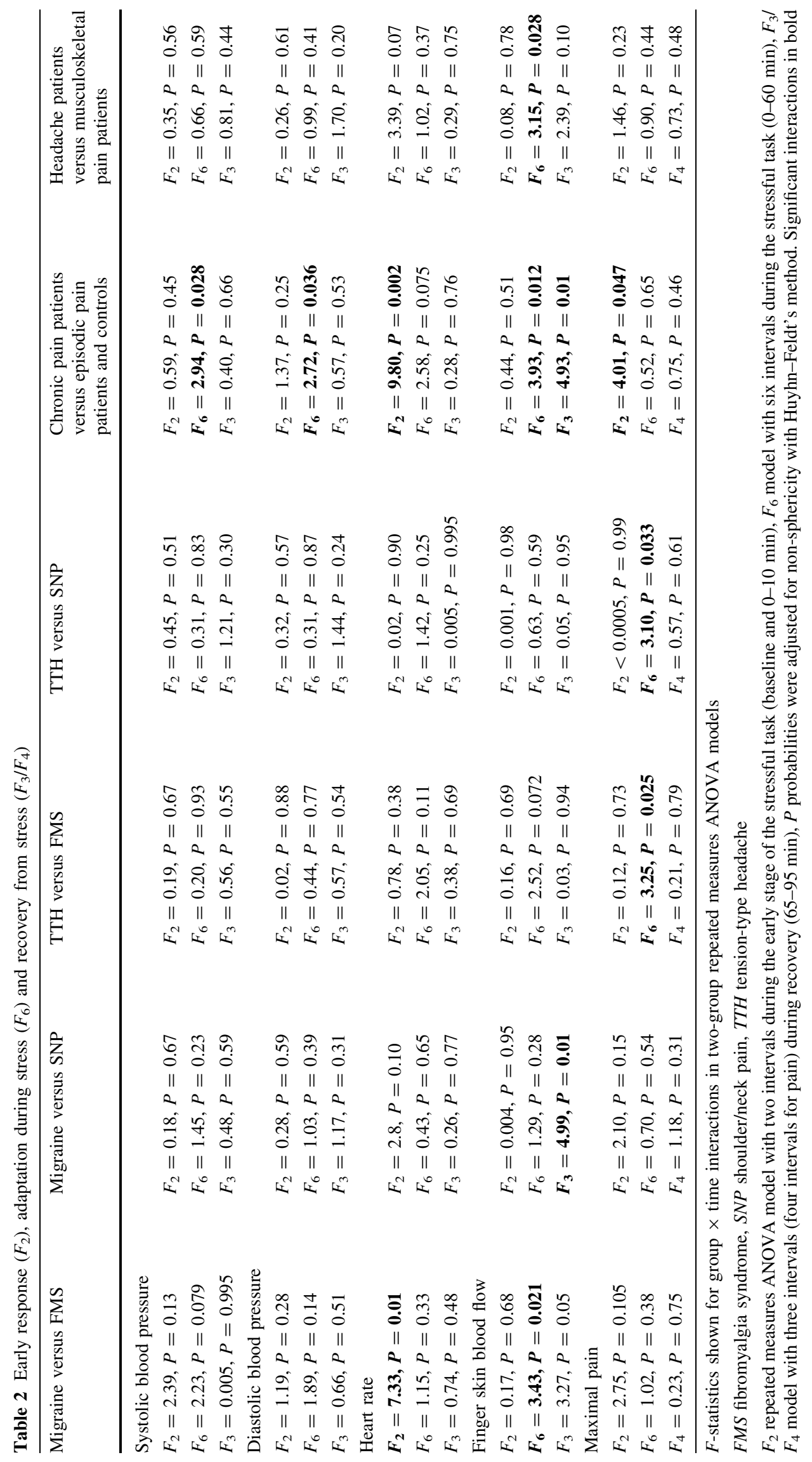


Chronic pain patients versus controls and migraineurs

We also made a comparison of the patients with chronic pain, i.e. FMS, SNP and the 12 TTH patients with chronic pain (Table 1), compared to non-chronic subjects (migraine, episodic TTH and controls grouped together; Fig. 2a-e). SBP and DBP profiles increased more throughout the test and there was more increase in vasoconstriction (i.e. less skin $\mathrm{BF}$ ) in chronic pain patients compared to non-chronic subjects $\left(F_{6} \geq 2.72, P \leq 0.036\right.$; Table 2). There was also less increase in the early HR response $(F=9.8, P=0.002)$ and the HR adaptation profile tended to decrease less throughout the stressful task for chronic pain patients $\left(F_{6}=2.58, P=0.075\right.$, Fig. $2 \mathrm{a}-$ d). Chronic pain patients also had a less increasing $F_{2}$ pain profile during the early phase of the stressful task ( $F=4.01, P=0.047$; Fig. 2e).

When investigating recovery from stress, chronic pain patients had a significantly less BF recovery compared to non-chronic subjects $\left(F_{3}=4.93, P=0.01\right.$; Table 2; Fig. 2d). BP and HR profiles were not different.

Headache patients versus patients with musculoskeletal pain

When we compared headache patients and musculoskeletal pain patients, a significantly larger increase in vasoconstriction was found in musculoskeletal pain patients compared to headache patients during the test $\left(F_{6}=3.15\right.$, $P=0.028$; Table 2). In addition, musculoskeletal pain patients had a trend towards less increase in HR during the first $10 \mathrm{~min}$ of the stressful task compared to headache patients $\left(F_{2}=3.39, P=0.07\right)$.

\section{Discussion}

The present study is to our knowledge the first to compare cardiovascular and pain responses and recovery during and after low-grade cognitive stress in FMS and TTH patients. It is of interest that no significant differences in BP, HR or $\mathrm{BF}$ responses to a mental stressor or recovery thereafter were found between TTH and FMS patients. In contrast, less increase in vasoconstriction during the stressful task was found in migraineurs compared to FMS patients. FMS patients also had a significantly lower early HR response compared to migraine patients. The similarities between TTH, FMS and SNP are interesting, as are the differences between these patients and migraine patients. Headaches and chronic musculoskeletal pain disorders are comorbid conditions [43], and a few earlier studies also suggest that TTH and FMS share some pathophysiological characteristics, but also that there are differences in the two diagnoses [6, 7]. Most of the studies have focused on pain physiology and muscle activity, as well as clinical features and epidemiological aspects.

The BF recovery in TTH, FMS and SNP patients all differed from migraine patients, suggesting that a common mechanism may explain at least the relative lack of $\mathrm{BF}$ recovery in these patients compared to migraine patients and controls [32, 34]. BP profiles were also similar among TTH, FMS and SNP patients, but they were not significantly different from those of migraineurs, although the curves in Fig. 1a-b appeared to be different.

The early HR response in FMS patients was different from that of migraineurs, and our group has previously also reported that the early $\mathrm{HR}$ response in FMS patients are different compared to controls [34]. A reduced HR response to stress in FMS patients has also been reported in several other studies [44-49], and some studies suggest a connection between a reduced stress response and the hyperalgesic state in FMS patients [29, 34, 50, 51]. While TTH and SNP were not statistically different from either migraine or FMS [32, 34] with regard to the early $\mathrm{HR}$ response (Table 2), there was a significant difference in the early HR response between chronic and non-chronic/healthy subjects (Fig. 2c; Table 2) suggesting that TTH and SNP are similar to FMS. We have previously suggested that TTH patients have a reduced or "blunted" response to stress, evident by a lack of an initial "spiked" response and followed by a more or less constant HR throughout the stress period [32]. However, our results indicate that there is no general relation between a low HR stress response and pain, as it is only the FMS patients who have a significant correlation between the $\mathrm{HR}$ response and pain development during the stressful task [34].

FMS patients had more vasoconstriction in skin blood vessels compared to migraineurs, both during and after the stressful task, while SNP (and TTH [32]) had more vasoconstriction only during the recovery period. One may speculate that this is a pain-induced phenomenon, as all three groups had more pain than the migraine patients, but no clear correlation between pain and BF was found (results not shown). Sympathetic dysregulation of skin BF would be an alternative hypothesis, for instance through central autonomic hyperactivity or hypersensitivity. However, as sympathetic activity influences BP, HR and BF, it is difficult to understand why skin BF should differ more between these groups than the other cardiovascular variables. A possible reason may be that while BF regulation in the finger skin is mediated mainly by sympathetic activity [52], BP and HR are mediated in a more complex way by both sympathetic and parasympathetic activity [53]. In addition, it is known that autonomic vasomotor activity in different organs is regulated individually, according to the functional needs of each organ [54-56]. 

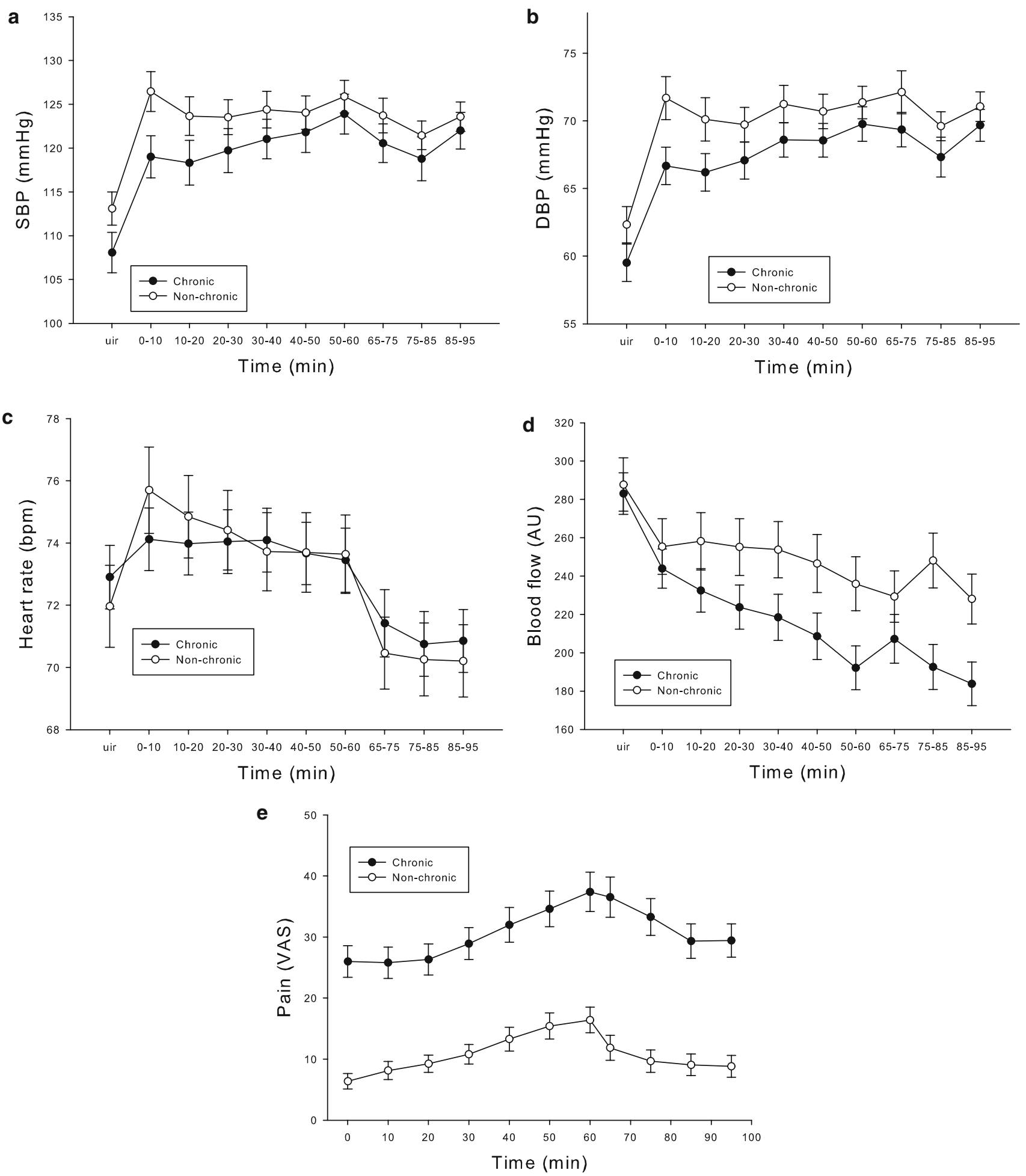

Fig. 2 Development of systolic BP (a), diastolic BP (b), heart rate (c) finger blood flow (d) and pain (e) throughout the stressful task and recovery period in chronic pain patients (chronic TTH, FMS and SNP) and episodic pain/no pain subjects (episodic TTH, migraine and

Although autonomic response profiles were similar on comparing TTH, FMS and SNP patients, TTH patients had a much higher increase in pain during the test than both controls). Values are given as group means (SEM). UIR: uninstructed rest period (baseline EMG). 0-60 min: duration of the cognitive stressful task. 65-95 min: relaxation period after the test

FMS and SNP patients, the latter groups being comparable to migraine patients and controls in this respect [17]. The larger increase in pain observed in TTH patients compared 
to the other patient groups may be interpreted as a "pain potentiation" reflecting increased temporal summation of pain, which is normally associated with central sensitisation. Central sensitisation has previously been suggested to be important not only in TTH [57-63], FMS and SNP [16, 64, 65], but also in migraine [66-68]. Our study suggests that TTH patients respond to stress with a much higher increase in pain than the other patients, but we have no data to determine whether this is due to differences in pain processing at a central or peripheral level.

On performing the same statistical analyses for chronic pain patients versus non-chronic subjects, as we did for the individual subject groups, we found differences between the groups that were not apparent when analysing differences between diagnostic groups. First of all, SBP, DBP and $\mathrm{BF}$ profiles during the stressful task were all different in chronic pain patients compared to migraineurs and controls, with the BP early responses being somewhat lower and continuing to increase throughout the test in the chronic group. Finger BF during the stressful task decreased more (i.e. more vasoconstriction) in the chronic group compared to the non-chronic group. Differentiating subjects by pain location (headache vs. musculoskeletal pain) did not provide any results not already found during the primary analyses on individual diagnoses. This indicates that subdividing the patients into groups based on the temporal pain patterns (chronic pain vs. episodic pain) results in a more homogenous group regarding the physiological responses to and recovery from low-grade cognitive stress, than if the subdivision is made based on anatomical pain location. This suggests that chronic pain patients have some stress-induced autonomic features in common.

In the recovery period, FMS and SNP patients had more vasoconstriction than migraine patients. Previously, we had reported increased vasoconstriction in TTH patients compared to migraine patients in the recovery period after stress [32]. Chronic pain patients also continued to have decrease in $\mathrm{BF}$ during the recovery period, while the episodic pain/control group seemed to stabilise. This finding is especially interesting in conjunction with the theoretical models of Eriksen and Ursin [19, 20] and McEwen [21, 22]. Both these models focus on subjects' inability to turn off a stress response, i.e. a lack of recovery, as a cause of health complaints. Repeated inability to recover from stress, for instance caused by the subject's inability to cope with the demands in his/her working environment, may create a vicious circle resulting in pain or other health complaints in some subjects. This model is supported by a study of Sluiter et al., who reported a connection between the need for recovery from work-related fatigue (presumably caused by stress and other work-environmental factors) and subjective health complaints [69]. TTH, FMS and SNP patients are often considered to be more sensitive to stress than healthy people and may thus be unable to mount a successful defence mechanism against stress, resulting in increased pain and prolonged physiological responses as seen from the reduced BF recovery among these patients in the present study. However, it must be emphasised that the present study does not provide evidence for a causal relation between the lack of $\mathrm{BF}$ recovery and pain development, but rather that these patients have an inability to recover from stress.

In conclusion, analyses of groups differentiated by pain chronicity indicated that cardiovascular stress responses, such as BP, HR and BF development, are similar among chronic TTH, FMS and SNP patients and different from that of migraine patients, episodic TTH patients and healthy controls, both during and after a stressful task. This suggests that these three chronic pain conditions may share some physiological mechanisms. Whether these mechanisms are causally related to pain, or just reflections of the chronic pain state, cannot be determined from the present study. As this is the first study that compares these patient groups in a long-lasting stress model, the results need confirmation from studies with similar methods. If confirmed, these findings may provide deeper insight into pathophysiological mechanisms and may improve treatment or prevention of these very prevalent and costly disorders.

Conflict of interest None.

\section{References}

1. Bergman S, Herrstrom P, Hogstrom K, Petersson IF, Svensson B, Jacobsson LT (2001) Chronic musculoskeletal pain, prevalence rates, and sociodemographic associations in a Swedish population study. J Rheumatol 28:1369-1377

2. Stovner LJ, Hagen K, Jensen R, Katsarava Z, Lipton RB, Scher AI, Steiner TJ, Zwart JA (2007) The global burden of headache: a documentation of headache prevalence and disability worldwide. Cephalalgia 27:193-210

3. Wolfe F, Ross K, Anderson J, Russell IJ, Hebert L (1995) The prevalence and characteristics of fibromyalgia in the general population. Arthritis Rheum 38:19-28

4. Aaron LA, Buchwald D (2001) A review of the evidence for overlap among unexplained clinical conditions. Ann Intern Med 134:868-881

5. Aaron LA, Burke MM, Buchwald D (2000) Overlapping conditions among patients with chronic fatigue syndrome, fibromyalgia, and temporomandibular disorder. Arch Intern Med 160:221-227

6. Schoenen J (2004) Tension-type headache and fibromyalgia: what's common, what's different? Neurol Sci 25(Suppl 3):S157S159

7. Lenaerts ME, Gill PS (2006) At the crossroads between tensiontype headache and fibromyalgia. Curr Pain Headache Rep 10:463-466 
8. McFarlane AC (2007) Stress-related musculoskeletal pain. Best Prac Res Clin Rheumatol 21:549-565

9. Nash JM, Thebarge RW (2006) Understanding psychological stress, its biological processes, and impact on primary headache. Headache 46:1377-1386

10. Bongers PM, Kremer AM, ter Laak J (2002) Are psychosocial factors, risk factors for symptoms and signs of the shoulder, elbow, or hand/wrist?: a review of the epidemiological literature. Am J Ind Med 41:315-342

11. Linton SJ (2000) A review of psychological risk factors in back and neck pain. Spine 25:1148-1156

12. van der Windt DA, Thomas E, Pope DP, de Winter AF, Macfarlane GJ, Bouter LM, Silman AJ (2000) Occupational risk factors for shoulder pain: a systematic review. Occup Environ Med 57:433-442

13. Davis MC, Zautra AJ, Reich JW (2001) Vulnerability to stress among women in chronic pain from fibromyalgia and osteoarthritis. Ann Behav Med 23:215-226

14. Vasseljen OJ, Westgaard RH (1996) Can stress-related shoulder and neck pain develop independently of muscle activity? Pain 64:221-230

15. Holte KA, Westgaard RH (2002) Daytime trapezius muscle activity and shoulder-neck pain of service workers with work stress and low biomechanical exposure. Am J Ind Med 41:393405

16. Nilsen KB, Westgaard RH, Stovner LJ, Helde G, Ro M, Sand TH (2006) Pain induced by low-grade stress in patients with fibromyalgia and chronic shoulder/neck pain, relation to surface electromyography. Eur J Pain 10:615-627

17. Leistad RB, Sand T, Westgaard R, Nilsen KB, Stovner LJ (2006) Stress-induced pain and muscle activity in patients with migraine and tension-type headache. Cephalalgia 26:64-73

18. Melin B, Lundberg U (1997) A biopsychosocial approah to workstress and musculoskeletal disorders. J Psychophysiol 11:238247

19. Eriksen HR, Ursin H (2002) Sensitization and subjective health complaints. Scand J Psychol 43:189-196

20. Ursin H, Eriksen HR (2004) The cognitive activation theory of stress. Psychoneuroendocrinology 29:567-592

21. McEwen BS (1998) Protective and damaging effects of stress mediators. N Engl J Med 338:171-179

22. McEwen BS, Stellar E (1993) Stress and the individual. Mechanisms leading to disease. Arch Intern Med 153:2093-2101

23. Brosschot JF, Pieper S, Thayer JF (2005) Expanding stress theory: prolonged activation and perseverative cognition. Psychoneuroendocrinology 30:1043-1049

24. van Amelsvoort LG, Kant IJ, Bultmann U, Swaen GM (2003) Need for recovery after work and the subsequent risk of cardiovascular disease in a working population. Occup Environ Med 60(Suppl 1):i83-87

25. de Croon EM, Sluiter JK, Frings-Dresen MH (2003) Need for recovery after work predicts sickness absence: a 2-year prospective cohort study in truck drivers. J Psychosom Res 55:331-339

26. Harris A, Ursin H, Murison R, Eriksen HR (2007) Coffee, stress and cortisol in nursing staff. Psychoneuroendocrinology 32:322330

27. Nilsen KB, Sand T, Stovner LJ, Leistad RB, Westgaard RH (2007) Autonomic and muscular responses and recovery to onehour laboratory mental stress in healthy subjects. BMC Musculoskelet Disord 8:81

28. Kristenson M, Eriksen HR, Sluiter JK, Starke D, Ursin H (2004) Psychobiological mechanisms of socioeconomic differences in health. Soc Sci Med 58:1511-1522

29. Okifuji A, Turk DC (2002) Stress and psychophysiological dysregulation in patients with fibromyalgia syndrome. Appl Psychophysiol Biofeedback 27:129-141
30. Wittrock DA, Myers TC (1998) The comparison of individuals with recurrent tension-type headache and headache-free controls in physiological response, appraisal, and coping with stressors: a review of the literature. Ann Behav Med 20:118-134

31. Petzke F, Clauw DJ (2000) Sympathetic nervous system function in fibromyalgia. Curr Rheumatol Rep 2:116-123

32. Leistad RB, Sand T, Nilsen KB, Westgaard RH, Stovner LJ (2007) Cardiovascular responses to cognitive stress in patients with migraine and tension-type headache. BMC Neurol 7:23

33. Leistad RB, Stovner LJ, White LR, Nilsen KB, Westgaard R, Sand $T$ (2007) Biochemical responses to stress in migraine and tension-type headache. J Headache Pain 8:157-166

34. Nilsen KB, Sand T, Westgaard RH, Stovner LJ, White LR, Leistad RB, Helde G, Rø M (2007) Autonomic activation and pain in response to low-grade mental stress in fibromyalgia and shoulder/neck pain patients. Eur J Pain 11(7):743-755

35. Headache Classification Committee otIHS (1988) Classification and diagnostic criteria for headache disorders, cranial neuralgias and facial pain. Headache Classification Committee of the International Headache Society. Cephalalgia 8(Suppl 7):1-96

36. Wolfe F, Smythe HA, Yunus MB, Bennett RM, Bombardier C, Goldenberg DL, Tugwell P, Campbell SM, Abeles M, Clark P (1990) The American College of Rheumatology 1990 Criteria for the Classification of Fibromyalgia. Report of the Multicenter Criteria Committee. Arthritis Rheum 33:160-172

37. Imholz BP, Langewouters GJ, van Montfrans GA, Parati G, van Goudoever J, Wesseling KH, Wieling W, Mancia G (1993) Feasibility of ambulatory, continuous 24-hour finger arterial pressure recording. Hypertension 21:65-73

38. Bansevicius D, Westgaard RH, Jensen C (1997) Mental stress of long duration: EMG activity, perceived tension, fatigue, and pain development in pain-free subjects. Headache 37:499-510

39. Westgaard RH, Bjørklund R (1987) Generation of muscle tension additional to postural muscle load. Ergonomics 30:911-923

40. Waersted M, Bjørklund RA, Westgaard RH (1994) The effect of motivation on shoulder-muscle tension in attention-demanding tasks. Ergonomics 37:363-376

41. Perneger TV (1998) What's wrong with Bonferroni adjustments. BMJ 316:1236-1238

42. Schulz KF, Grimes DA (2005) Multiplicity in randomised trials I: endpoints and treatments. Lancet 365:1591-1595

43. Hagen K, Einarsen C, Zwart JA, Svebak S, Bovim G (2002) The co-occurrence of headache and musculoskeletal symptoms amongst 51050 adults in Norway. Eur J Neurol 9:527-533

44. van Denderen JC, Boersma JW, Zeinstra P, Hollander AP, van Neerbos BR (1992) Physiological effects of exhaustive physical exercise in primary fibromyalgia syndrome (PFS): is PFS a disorder of neuroendocrine reactivity? Scand J Rheumatol 21:35-37

45. Martínez-Lavín M, Hermosillo AG, Mendoza C, Ortiz R, Cajigas JC, Pineda C, Nava A, Vallejo M (1997) Orthostatic sympathetic derangement in subjects with fibromyalgia. J Rheumatol 24:714718

46. Bou-Holaigah I, Calkins H, Flynn JA, Tunin C, Chang HC, Kan JS, Rowe PC (1997) Provocation of hypotension and pain during upright tilt table testing in adults with fibromyalgia. Clin Exp Rheumatol 15:239-246

47. Kelemen J, Láng E, Bálint G, Trócsányi M, Müller W (1998) Orthostatic sympathetic derangement of baroreflex in patients with fibromyalgia. J Rheumatol 25:823-825

48. Cohen H, Neumann L, Alhosshle A, Kotler M, Abu-Shakra M, Buskila D (2001) Abnormal sympathovagal balance in men with fibromyalgia. J Rheumatol 28:581-589

49. Cohen H, Neumann L, Shore M, Amir M, Cassuto Y, Buskila D (2000) Autonomic dysfunction in patients with fibromyalgia: application of power spectral analysis of heart rate variability. Semin Arthritis Rheum 29:217-227 
50. Clauw DJ, Chrousos GP (1997) Chronic pain and fatigue syndromes: overlapping clinical and neuroendocrine features and potential pathogenic mechanisms. Neuroimmunomodulation 4:134-153

51. Adler GK, Kinsley BT, Hurwitz S, Mossey CJ, Goldenberg DL (1999) Reduced hypothalamic-pituitary and sympathoadrenal responses to hypoglycemia in women with fibromyalgia syndrome. Am J Med 106:534-543

52. Franchini KG, Cowley AWJ (2004) Neurogenic control of blood vessels. In: Robertson D (ed) Primer on the autonomic nervous system. Elsevier Academic Press, London, pp 139-143

53. Franchini KG, Cowley AWJ (2004) Autonomic control of cardiac function. In: Robertson D (ed) Primer on the autonomic nervous system. Elsevier Academic Press, London, pp 134-138

54. Jänig W, Häbler HJ (2003) Neurophysiological analysis of targetrelated sympathetic pathways-from animal to human: similarities and differences. Acta Physiol Scand 177:255-274

55. Sved AF, Cano G, Card JP (2001) Neuroanatomical specificity of the circuits controlling sympathetic outflow to different targets. Clin Exp Pharmacol Physiol 28:115-119

56. Gibbins IL, Jobling P, Morris JL (2003) Functional organization of peripheral vasomotor pathways. Acta Physiol Scand 177:237245

57. Ashina M (2004) Neurobiology of chronic tension-type headache. Cephalalgia 24:161-172

58. Ashina S, Bendtsen L, Ashina M (2005) Pathophysiology of tension-type headache. Curr Pain Headache Rep 9:415-422

59. Ashina S, Bendtsen L, Ashina M, Magerl W, Jensen R (2006) Generalized hyperalgesia in patients with chronic tension-type headache. Cephalalgia 26:940-948
60. Bendtsen L (2000) Central sensitization in tension-type headache-possible pathophysiological mechanisms. Cephalalgia 20:486-508

61. Bendtsen L (2003) Central and peripheral sensitization in tensiontype headache. Curr Pain Headache Rep 7:460-465

62. Jensen R (1999) Pathophysiological mechanisms of tension-type headache: a review of epidemiological and experimental studies. Cephalalgia 19:602-621

63. Jensen R, Olesen J (1996) Initiating mechanisms of experimentally induced tension-type headache. Cephalalgia 16:175-182; discussion 138-179

64. Price DD, Staud R (2005) Neurobiology of fibromyalgia syndrome. J Rheumatol Suppl 75:22-28

65. Staud R, Rodriguez ME (2006) Mechanisms of disease: pain in fibromyalgia syndrome. Nature Clin Pract 2:90-98

66. Burstein R (2001) Deconstructing migraine headache into peripheral and central sensitization. Pain 89:107-110

67. Malick A, Burstein R (2000) Peripheral and central sensitization during migraine. Funct Neurol 15(Suppl 3):28-35

68. Yamamura H, Malick A, Chamberlin NL, Burstein R (1999) Cardiovascular and neuronal responses to head stimulation reflect central sensitization and cutaneous allodynia in a rat model of migraine. J Neurophysiology 81:479-493

69. Sluiter JK, de Croon EM, Meijman TF, Frings-Dresen MH (2003) Need for recovery from work related fatigue and its role in the development and prediction of subjective health complaints. Occup Environ Med 60(Suppl 1):i62-70 